\title{
Fatores prognósticos para o desenvolvimento de metástases nos sarcomas de tecidos moles"
}

\author{
Prognosis factors in the development \\ of metastases in soft tissue sarcomas
}

\begin{abstract}
Luiz Eduardo Moreira Teixeira ${ }^{1}$, Ivana Duval Araújo2 ${ }^{2}$ Marco Antônio Percope de Andrade ${ }^{3}$, Paulo Guilherme de Oliveira Salles ${ }^{4}$, Ricardo Horta Miranda ${ }^{5}$, Daniel Ferreira Ghedini ${ }^{6}$, Gustavo Albergaria de MAgalHÃEs ${ }^{6}$
\end{abstract}

\section{RESUMO}

Objetivo: Determinar os fatores prognósticos relacionados com o desenvolvimento de metástases a distância nos pacientes com diagnóstico de sarcoma de tecidos moles (STM) em extremidades. Méto-

* Trabalho realizado no Serviço de Ortopedia e Traumatologia do Hospital das Clínicas da Universidade Federal de Minas Gerais UFMG - Belo Horizonte (MG), Brasil e no Biocor Instituto UFMG - Belo Horizonte (MG), Brasil. Parte da dissertação apresentada ao Curso de Pós-graduação em Cirurgia pela Universidade Federal de Minas Gerais para obtenção do título de Mestre.

1. Mestre, Coordenador do Serviço de Oncologia Ortopédica do Hospital das Clínicas da Universidade Federal de Minas Gerais UFMG - Belo Horizonte (MG), Brasil.

2. Doutor, Professor Associado do Departamento de Cirurgia da Faculdade de Medicina da Universidade Federal de Minas Gerais UFMG - Belo Horizonte (MG), Brasil.

3. Doutor, Professor Adjunto do Departamento do Aparelho Locomotor da Faculdade de Medicina da Universidade Federal de Minas Gerais - UFMG - Belo Horizonte (MG), Brasil.

4. Mestre, Patologista do Laboratório Dairton Miranda - Belo Horizonte (MG), Brasil.

5. Coordenador do Serviço de Oncologia Ortopédica da Santa Casa de Belo Horizonte (MG), Brasil.

6. Residente do Serviço de Ortopedia e Traumatologia do Hospital das Clínicas da Universidade Federal de Minas Gerais - UFMG - Belo Horizonte (MG), Brasil.

Endereço para correspondência: Luiz Eduardo M. Teixeira, Av. do Contorno, 7.485, Bairro Santo Antônio - 30110-120 - Belo Horizonte, MG. E-mail: luizmteixeira@yahoo.com.br

Recebido em 10/4/08. Aprovado para publicação em 13/5/08.

Copyright RBO2008 dos: Foram avaliados 30 pacientes tratados por ressecção cirúrgica de STM, com seguimento médio de $36,5 \pm 12,2$ meses, utilizando como fatores prognósticos: idade, sexo, localização, profundidade, localização em compartimentos anatômicos, tamanho, manipulação prévia ao tratamento definitivo, margens cirúrgicas, grau de malignidade histológica, presença de necrose e invasão vascular à histologia. Esses fatores foram correlacionados com o desenvolvimento de metástases por análise univariada e multivariada, sendo considerados significativos valores de $p \leq 0,05$. Resultados: Na análise univariada houve correlação do desenvolvimento de metástases com a localização extracompartimental ( $p$ $=0,002)$, com o tamanho $>10 \mathrm{~cm}(\mathrm{p}=0,007)$, com o alto grau de malignidade $(p=0,007)$, com a presença de necrose $(p=0,002)$ e com a presença de invasão vascular $(p=0,034)$. A idade $(p=1,000)$, o sexo $(0,709)$, a localização em segmentos corporais $(p=$ $\mathbf{0 , 2 9 8})$, a profundidade $(p=0,288)$, a margem cirúrgica $(p=0,419)$ e a manipulação prévia do tumor $(p=1,000)$ não apresentaram correlação com a ocorrência de metástases a distância. Na análise multivariada apenas a localização extracompartimental $(p=0,008), o$ tamanho $(p=0,018)$ e a presença de invasão vascular $(p=0,043)$ foram significativos. Conclusão: O desenvolvimento de metástases a distância nos pacientes com sarcoma de tecidos moles depende da localização extracompartimen- 


\section{tal, do tamanho $(>10 \mathrm{~cm})$, do alto grau de maligni- dade histológica, da presença de necrose e da inva- são vascular à histologia.}
Descritores - Neoplasias de tecidos moles, Prognóstico; Me- tástase neoplásica

\section{ABSTRACT}

Objective: To determine the prognostic factors related to the development of remote metastases in patients with the diagnosis of soft tissue sarcoma (STS) in the extremities. Methods: 30 patients treated with surgical resection of STS with a mean follow-up of $36.5 \pm 12.2$ months were evaluated using the following prognosis factors: age, gender, location, depth, location in anatomic compartments, size, handling before final treatment, surgical borders, degree of histological malignancy, presence of necrosis, and histologically-verified vascular invasion. Such factors were correlated to the development of metastases by univariate analysis, values of $p \leq 0.05$ being considered significant. Results: The univariate analysis showed a correlation of the development of metastases and the extra-compartmental location $(p=0.002)$, size $>10$ $\mathrm{cm}(p=0.007)$, the high degree of malignancy $(p=$ $0.007)$, the presence of necrosis $(p=0.002)$ and the presence of vascular invasion $(p=0.034)$. Age $(p=$ 1.000), gender (0.709), location in body segments ( $p$ $=0.298)$, depth $(p=0,288)$, surgical border $(p=$ $0.419)$, and prior handling of the tumor $(p=1.000)$ did not show a correlation with the occurrence of remote metastases. In the multivariate analysis, only the extra-compartmental location $(p=0.008)$, size ( $p$ $=0.018)$, and the presence of vascular invasion ( $p=$ 0.043) were significant. Conclusion: The development of remote metastases in patients with soft tissue sarcoma depends on the extracompartmental location, on the size (>10 cm), on the high degree of malignancy seen in histology, on the presence of necrosis, and on vascular invasion seen in histology.

\footnotetext{
Keywords - Soft tissue neoplasms; Prognosis; Neoplasm metastasis
}

\section{INTRODUÇÃO}

O termo sarcoma de tecidos moles (STM) define um grupo heterogêneo de neoplasias malignas extra-esqueléticas de origem mesenquimal ${ }^{(1-2)}$. São tumores relativamente raros e apresentam grande variedade de subtipos histológicos e distribuição pelo corpo, tornando difícil a obtenção de informações consistentes sobre a história natural, prognóstico e tratamento ${ }^{(2-3)}$.

O tratamento de escolha dos STM é a cirurgia, complementada ou não pela radioterapia e pela quimioterapia $^{(3)}$. A necessidade do tratamento quimioterápico adjuvante não é consenso na literatura e sua indicação depende de vários fatores prognósticos. A combinação desses fatores cria sistemas de estadiamento que pretendem separar os pacientes de acordo com a expectativa de evolução da doença ${ }^{(4)}$.

Vários fatores têm sido associados com o prognóstico nos STM, como idade, sexo, localização, profundidade, tamanho, tipo histológico, grau de malignidade histológica, necrose tumoral, invasão vascular à microscopia e aneuploidias. Alguns desses fatores são consensuais, enquanto outros não têm mostrado resultados consistentes ${ }^{(3-8)}$. Dentre esses fatores, os mais associados ao desenvolvimento de metástases são o alto grau de malignidade histológica, o tamanho inicial do tumor, a profundidade em relação à fáscia muscular e a presença de necrose e invasão vascular detectadas pela histologia ${ }^{(4-7)}$.

O objetivo deste estudo foi determinar os fatores associados ao desenvolvimento de metástases em STM localizados em extremidades.

\section{MÉTODOS}

No período entre janeiro de 2000 e novembro de 2005 foram atendidos no Serviço de Ortopedia e Traumatologia do Hospital das Clínicas da Universidade Federal de Minas Gerais (HC-UFMG) e no Biocor Instituto (BI) 42 pacientes com diagnóstico de sarcomas extra-esqueléticos localizados em extremidades. Foram excluídos do estudo 12 pacientes: três não apresentavam dados suficientes no prontuário, três perderam o seguimento clínico e seis apresentavam tumores de evolução ou tratamento diferentes, sendo eles o dermatofibrossarcoma $(n=3)$, rabdomiossarcoma $(n=$ 
TABELA 1

Diagnóstico dos tipos histológicos dos STM de 30 pacientes submetidos à ressecção cirúrgica no Hospital das Clínicas da UFMG e no Biocor Instituto entre janeiro de 2000 e novembro de 2005

\begin{tabular}{lccc}
\hline \multicolumn{1}{c}{ Diagnóstico } & $\begin{array}{c}\text { Número de } \\
\text { pacientes (n) }\end{array}$ & $\begin{array}{c}\text { Freqüência } \\
\text { relativa (\%) }\end{array}$ & $\begin{array}{c}\text { Freqüência } \\
\text { acumulada (\%) }\end{array}$ \\
\hline Fibrohistiocitoma maligno & 7 & 23,3 & 23,3 \\
Sinoviossarcoma & 7 & 23,3 & 46,6 \\
Lipossarcoma & 4 & 13,3 & 59,9 \\
Fibrossarcoma & 2 & 6,7 & 66,7 \\
Leiomiossarcoma & 2 & 6,7 & 73,4 \\
Neurofibrossarcoma & 2 & 6,7 & 80,1 \\
Sarcoma epitelióide & 2 & 6,7 & 86,8 \\
Angiossarcoma & 1 & 3,3 & 90,1 \\
Sarcoma de células claras & 1 & 3,3 & 93,4 \\
Hemangiopericitoma maligno & 1 & 3,3 & 96,7 \\
Sarcoma de origem indeterminada & 1 & 3,3 & 100 \\
Total & 30 & 100 & 100
\end{tabular}

1), tumor de Ewing extra-esquelético $(n=1)$ e linfoma não-Hodgkin $(n=1)$. A média de idade dos pacientes foi de 47,66 \pm 19,1 anos, variando entre 18 e 86 anos. O tempo médio de acompanhamento foi de $36,5 \pm 12,2$ meses, com o mínimo de 19 meses e o máximo de 62 meses. Dezoito (60\%) eram do sexo masculino e 12 (40\%) do feminino. O diagnóstico histológico está listado na tabela 1 . Todos os pacientes foram submetidos a exame tomográfico ou de ressonância magnética do segmento acometido, tomografia computadorizada de tórax e abdome e cintilografia óssea de corpo total. O diagnóstico foi confirmado por meio de biópsia incisional aberta para estudo histopatológico, complementado pelo perfil imuno-histoquímico quando necessário. Os pacientes foram estadiados de acordo com Enneking et al ${ }^{(9)}$ (tabela 2).

A cirurgia consistiu de uma ressecção em bloco da lesão incluindo o tecido normal que envolvia o tumor, o trajeto prévio da biópsia e o orifício de saída do dreno quando presente. A amputação foi indicada para tumores volumosos, agressivos, quando margem de ressecção adequada não foi possível, nos casos infectados e quando não houve cobertura adequada de partes moles. Dos 30 pacientes da amostra, 24 (80\%) foram submetidos à cirurgia conservadora e seis (20\%), a amputações. Nos pacientes encaminhados após biópsia ou ressecção prévia, foi realizada a ressecção do
TABELA 2

\begin{tabular}{|c|c|c|c|}
\hline \multicolumn{4}{|c|}{$\begin{array}{l}\text { Estadiamento de acordo com a classificação proposta } \\
\text { por Enneking et al(9), dos } 30 \text { pacientes submetidos } \\
\text { ao tratamento cirúrgico no Hospital das Clínicas } \\
\text { da UFMG e no Biocor Instituto no período de } \\
\text { janeiro de } 2000 \text { e novembro de } 2005\end{array}$} \\
\hline $\begin{array}{c}\text { nneking } \\
\text { et al }\end{array}$ & $\begin{array}{l}\text { Número de } \\
\text { pacientes (n) }\end{array}$ & $\begin{array}{l}\text { Freqüência } \\
\text { relativa (\%) }\end{array}$ & $\begin{array}{c}\text { Freqüência } \\
\text { acumulada (\%) }\end{array}$ \\
\hline IA & 7 & 23,3 & 23,3 \\
\hline IB & 4 & 13,3 & 36,6 \\
\hline IIA & 5 & 16,6 & 53,3 \\
\hline IIB & 10 & 33,3 & 86,7 \\
\hline III & 4 & 13,3 & 100 \\
\hline Total & 30 & 100 & 100 \\
\hline
\end{tabular}

tumor residual ou a ampliação das margens, após revisão do estudo histopatológico e do estadiamento local e sistêmico.

As peças cirúrgicas foram enviadas para estudo histopatológico para definição dos seguintes parâmetros: confirmação do diagnóstico de sarcoma, margens cirúrgicas, grau de malignidade, presença de necrose e invasão vascular. O estudo foi revisto por um único patologista para padronização dos achados histológicos.

O tratamento adjuvante baseou-se em decisão multidisciplinar utilizando como parâmetros o tamanho, a agressividade, a presença de metástases e as margens 
operatórias, e cada tratamento era individualizado, sem um protocolo específico. A radioterapia adjuvante foi realizada em 19 (63,3\%) pacientes, sempre no período pós-operatório. A quimioterapia adjuvante foi realizada em $18(60 \%)$ pacientes.

O controle pós-operatório consistiu de exame clínico, tomografia de tórax, cintilografia óssea e ressonância magnética do segmento operado. Em caso de recidiva ou metástases, os pacientes eram submetidos ao tratamento complementar.

Foram avaliados como fatores prognósticos: a idade ( $<50$ anos ou $\geq 50$ anos), o sexo, a localização por segmento corporal (membros superiores, membros inferiores e pelve), a localização em compartimentos (intra ou extracompartimentais), a profundidade em relação à fáscia muscular (profundos e superficiais), o tamanho do tumor $(\leq 10 \mathrm{~cm}$ ou $>10 \mathrm{~cm})$, a manipulação prévia ao tratamento definitivo, as margens cirúrgicas (livres ou comprometidas), o grau de malignidade histológica (alto ou baixo grau), a presença de necrose e a invasão vascular detectadas pela histologia.

O estudo estatístico foi realizado por meio da análise univariada de associação entre a variável resposta (metástase) e cada uma das variáveis independentes utilizando tabelas tipo $2 \times 2$ ou $3 \times 2$ e aplicando-se o teste do qui-quadrado $\left(\chi^{2}\right)$ ou o teste exato de Fisher quando uma das frequiências era menor do que 5. Foram considerados significativos valores de $\mathrm{p} \leq 0,05$ $(5 \%)$. Após a análise univariada, os fatores relevantes $(\mathrm{p}<0,250)$ e as variáveis dependentes foram incluídos em análise multivariada por regressão logística múltipla. A proporção da variabilidade foi determinada pelo coeficiente de Nagelkerke e o método utilizado na regressão foi o stepwise backward. Curvas de sobrevida foram construídas para a sobrevida global e sobrevida livre de metástases, utilizando-se o método produto-limite de Kaplan-Meier. A análise foi realizada com o auxílio do software SPSS for Windows ${ }^{\circledR}$ (versão 12.0, Chicago, EUA).

O estudo foi aprovado pelo Comitê de Ética do HCUFMG e do BI, com aprovação final pelo Comitê de Ética em Pesquisa da Universidade Federal de Minas Gerais (parecer no ETIC 002/07).

\section{RESULTADOS}

Dos 30 pacientes incluídos no estudo, 12 (40\%) apresentaram metástase a distância (gráfico 1). A localização mais freqüente de disseminação metastática foi para

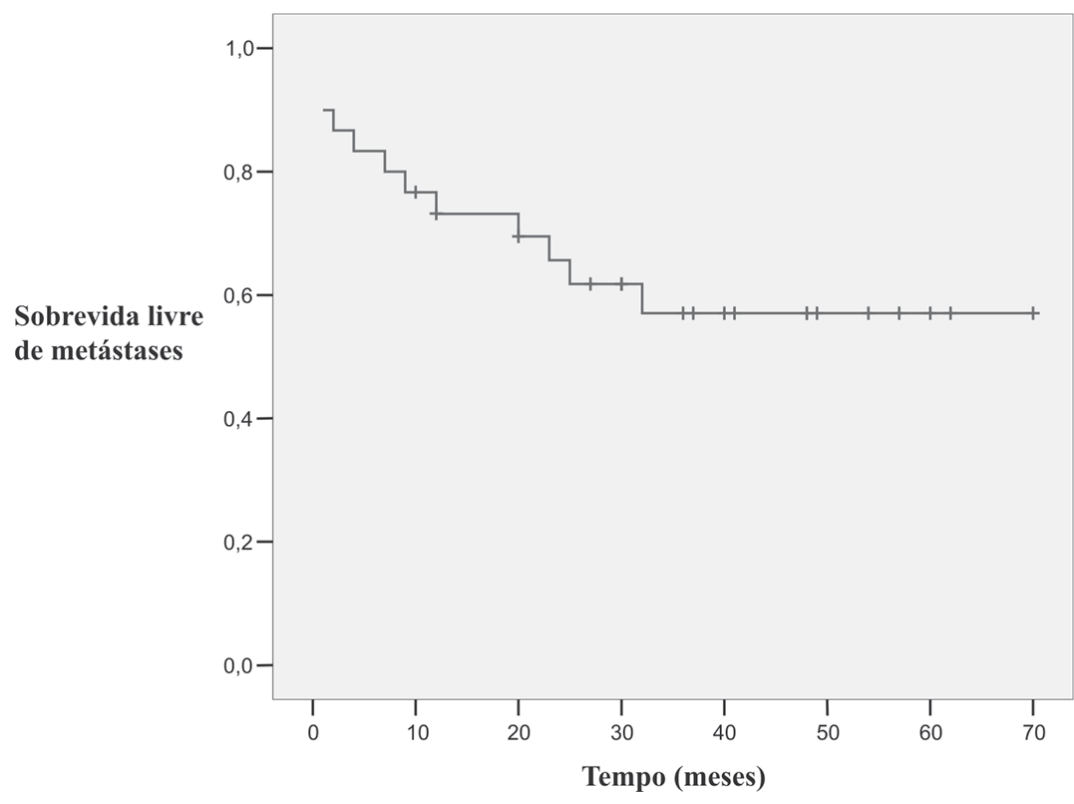

Gráfico 1 - Curva de sobrevida livre de metástases de 30 pacientes submetidos à ressecção cirúrgica de STM no Hospital das Clínicas e no Biocor Instituto no período de janeiro de 2000 a novembro de 2005 
o pulmão, observada em $11(36,7 \%)$ casos, seguida para os linfonodos, presente em três pacientes $(10 \%)$ em associação com metástases pulmonares; em dois $(6,6 \%)$ pacientes detectamos metástase óssea, sendo uma isolada e outra associada à metástase pulmonar.
Os resultados da análise univariada estão expressos na tabela 3. Dos pacientes com idade inferior a 50 anos, sete $(41,2 \%)$ apresentaram metástase e naqueles com idade igual ou superior a 50 anos ela foi observada em cinco $(38,5 \%)$ casos, não havendo diferença significa-

TABELA 3

Análise univariada para correlação dos fatores prognósticos e o desenvolvimento de metástases

\begin{tabular}{|c|c|c|c|c|c|}
\hline Fatores prognósticos & n (\%) & $\begin{array}{c}\text { Metástases } \\
\text { n (\%) }\end{array}$ & $\mathbf{p}$ & $\mathbf{R R}$ & IC (95\%) \\
\hline \multicolumn{6}{|l|}{ Idade } \\
\hline$<50$ anos & $17(56,7)$ & $7(41,2)$ & \multirow{2}{*}{1,000} & \multirow{2}{*}{0,87} & \multirow{2}{*}{$0,42-1,82$} \\
\hline$\geq 50$ anos & $13(43,3)$ & $5(38,5)$ & & & \\
\hline \multicolumn{6}{|l|}{ Sexo } \\
\hline Masculino & $18(60)$ & $8(44,4)$ & \multirow{2}{*}{0,709} & \multirow{2}{*}{2,18} & \multirow{2}{*}{$0,63-7,50$} \\
\hline Feminino & $12(40)$ & $4(33,3)$ & & & \\
\hline \multicolumn{6}{|l|}{ Localização } \\
\hline MMII & $20(66,7)$ & $9(45)$ & \multirow{3}{*}{0,298} & \multirow{3}{*}{-} & \multirow{3}{*}{-} \\
\hline MMSS & $7(23,3)$ & $3(42)$ & & & \\
\hline Pelve & $3(10)$ & $0 \quad(0)$ & & & \\
\hline \multicolumn{6}{|l|}{ Profundidade } \\
\hline Profundo & $27(90)$ & $12(44,4)$ & \multirow{2}{*}{0,224} & \multirow{2}{*}{-} & \multirow{2}{*}{-} \\
\hline Superficial & $3(10)$ & $0 \quad(0)$ & & & \\
\hline \multicolumn{6}{|l|}{ Compartimentos } \\
\hline Intracompartimental & $16(53,3)$ & $2(12,5)$ & \multirow{2}{*}{0,002} & \multirow{2}{*}{17,50} & \multirow{2}{*}{$2,66-114,84$} \\
\hline Extracompartimental & $14(46,7)$ & $10(71,4)$ & & & \\
\hline \multicolumn{6}{|l|}{ Tamanho } \\
\hline$\leq 10 \mathrm{~cm}$ & $12(40)$ & $1(8,3)$ & \multirow{2}{*}{0,007} & \multirow{2}{*}{17,28} & \multirow{2}{*}{$1,81-164,95$} \\
\hline$>10 \mathrm{~cm}$ & $18(60)$ & $11(61,1)$ & & & \\
\hline \multicolumn{6}{|l|}{ Margem cirúrgica } \\
\hline Livre & $22(73,4)$ & $10(45,5)$ & \multirow{2}{*}{0,419} & \multirow{2}{*}{0,40} & \multirow{2}{*}{$0,066-2,437$} \\
\hline Comprometida & $8(26,6)$ & $2(25,0)$ & & & \\
\hline \multicolumn{6}{|l|}{ Manipulação prévia } \\
\hline Sim & $18(60)$ & $7(38,9)$ & 1000 & 111 & $0.32-3.81$ \\
\hline Não & $12(40)$ & $5(41,7)$ & 1,000 & 1,11 & $0,0<-3,01$ \\
\hline Grau histológico & & & & & \\
\hline Alto & $18(40)$ & $11(61,1)$ & 0007 & 1728 & $181-16495$ \\
\hline Baixo & $12(60)$ & $1 \quad(8,3)$ & 0,001 & $1 /, 28$ & 1,81-164,95 \\
\hline Necrose histológica & & & & & \\
\hline Sim & $12(40)$ & $9(75)$ & 0,002 & 1500 & $247-9084$ \\
\hline Não & $18(60)$ & $3(16,7)$ & 0,002 & 10,00 & $2,4 /-90,04$ \\
\hline Invasão vascular histológ & & & & & \\
\hline Sim & $8(26,6)$ & $6(75)$ & & & \\
\hline Não & $22(73,4)$ & $6(27,3)$ & 0,034 & 8,00 & $1,25-51,13$ \\
\hline
\end{tabular}

MMSS: membros superiores; MMII: membros inferiores; RR: risco relativo; IC: intervalo de confiança. 
tiva entre os grupos $(\mathrm{p}=1,000)$. Em relação ao sexo, oito $(44,4 \%)$ pacientes do sexo masculino e quatro $(33,3 \%)$ do grupo feminino apresentaram metástases $(\mathrm{p}=0,709)$. Quando avaliada a correlação entre metástases e a localização dos tumores, foi observado que nove $(45 \%)$ pacientes com tumores localizados nos MMII e três (42\%) pacientes com tumores em MMSS desenvolveram metástases, não havendo significância estatística ( $\mathrm{p}=0,298)$. A profundidade também não foi significativa, apesar de os $12(44,4 \%)$ casos de metástases ocorrerem em lesões profundas $(p=0,224)$. Quanto à localização em compartimentos anatômicos, a metástase ocorreu em dois $(12,5 \%)$ pacientes com tumores intracompartimentais e em $10(71,4 \%)$ pacientes com tumores extracompartimentais, sendo a diferença significante $(p=0,002)$. Em relação ao tamanho do tumor, foram vistas metástases em um paciente $(8,3 \%)$ com tumor menor ou igual a $10 \mathrm{~cm}$ e em 11 pacientes $(61,1 \%)$ com tumores maiores do que $10 \mathrm{~cm}$, sendo a diferença estatisticamente significativa $(\mathrm{p}=$ 0,007). Nos pacientes com margens cirúrgicas comprometidas, houve dois casos $(25 \%)$ de metástases, enquanto nos com margens livres a ocorrência de metástases foi observada em $10(45,5 \%)$, não sendo a diferença significativa $(p=0,419)$. No grupo de pacientes submetidos à manipulação prévia do tumor, sete $(38,9 \%)$ evoluíram com metástases a distância e nos encaminhados para tratamento antes de qualquer procedimento operatório prévio, cinco $(41,7 \%)$ evoluíram com metástases $(\mathrm{p}=1,000)$.

Quando avaliados os parâmetros histológicos, 11 pacientes $(61,1 \%)$ com tumores de alto grau de malignidade apresentaram metástases, enquanto apenas um paciente $(8,3 \%)$ com tumor de baixo grau evoluiu com metástase, sendo a diferença significativa $(\mathrm{p}=$ $0,007)$. A presença de necrose tecidual observada na histologia apresentou correlação positiva com a ocorrência de metástases; nove pacientes (75\%) com necrose evoluíram com metástases em comparação com apenas três $(16,7 \%)$ dos que não apresentavam necrose $(\mathrm{p}=0,002)$. Nos pacientes com invasão vascular intratumoral, seis de um total de oito $(75 \%)$ desenvolveram metástases, mesmo número de pacientes $(27,3 \%)$ observado no grupo sem invasão vascular, sendo a diferença estatisticamente significativa $(\mathrm{p}=$ 0,034).

$\mathrm{Na}$ análise multivariada, o modelo selecionado apresentou $66 \%$ de coeficiente de Nagelkerke, $p=0,953$ na qualidade do ajuste, $94,4 \%$ de predição negativa e $75 \%$ de predição positiva. Nesse modelo, os fatores que foram considerados significativos foram a presença de invasão vascular à histologia $(p=0,073 ; R R=26,39)$, o tamanho do tumor maior de $10 \mathrm{~cm}(\mathrm{p}=0,018$; $\mathrm{RR}=$ $32,7)$ e a localização extracompartimental $(\mathrm{p}=0,008$; $\mathrm{RR}=31,1)$.

\section{DISCUSSÃO}

Os resultados do tratamento dos pacientes com STM têm mostrado pouco avanço nos últimos anos e a indicação do uso da quimioterapia adjuvante é controver$\mathrm{sa}^{(3-5)}$. Estudos iniciais mostraram melhora na sobrevida global e na sobrevida livre de doença, mas trabalhos subseqüentes não conseguiram comprovar o benefício da quimioterapia ${ }^{(10-11)}$. Somente pacientes com alto risco para desenvolver metástases devem ser submetidos ao tratamento adjuvante ${ }^{(12)}$. A inclusão de pacientes de baixo risco em regimes quimioterápicos tem a desvantagem de submetê-los a uma série de efeitos colaterais e de mascarar o real benefício do tratamento. Neste estudo, procurou-se analisar um grupo de pacientes submetidos a tratamento cirúrgico pela mesma equipe médica, na tentativa de validar os fatores prognósticos mais influentes no desenvolvimento de metástases, sendo as características da casuística como sexo, idade, subtipos e localização representativas dos pacientes com STM em geral. O tempo de seguimento foi também suficiente para detectar a ocorrência de metástases, já que a maioria destas ocorre nos dois primeiros anos de seguimento ${ }^{(13-14)}$.

A disseminação metastática foi observada em $40 \%$ dos casos, compatível com a literatura, que mostra incidência de metástases variando de $26,1 \%$ a $56,1 \%{ }^{(2-}$ $5,13,15)$. Os fatores determinantes foram o tamanho maior do que $10 \mathrm{~cm}$, a localização extracompartimental, o alto grau de malignidade histológica e a presença de necrose e invasão vascular detectadas pela histologia.

O tamanho do tumor é um importante fator prognóstico nos STM, tanto para disseminação metastática 
quanto para recorrência local ${ }^{(3-5,10-15)}$. Trovik et al observaram aumento no risco relativo de metástases de $1,5 \%$ para cada aumento de $5 \mathrm{~cm}$ no tamanho do tumor ${ }^{(16)}$. Entretanto, não há consenso no ponto de corte que deve ser utilizado para separar o grupo de alto ris$\mathrm{co}^{(3-5)}$. Os ensaios clínicos utilizam como ponto de corte o valor de $5 \mathrm{~cm}, 8 \mathrm{~cm}$ ou $10 \mathrm{~cm}^{(2-5)}$. Utilizamos o valor de corte de $10 \mathrm{~cm}$ para separar os pacientes em dois grupos por ser o que mais se aproxima do tamanho médio dos tumores de nossa casuística e por ser o mais usado na literatura.

A localização extracompartimental foi importante para a ocorrência de metástases. Os tumores extracompartimentais usualmente apresentam tratamento cirúrgico mais difícil e margens mais exíguas, já que se encontram em íntima correlação com estruturas neurovasculares. A maioria dos estudos não utiliza a localização em compartimentos como fator prognóstico isolado $^{(3,17-19)}$. Rööser et $a l^{(20)}$, avaliando o sistema de classificação de Enneking et $a l^{(9)}$, observaram que, embora os tumores considerados extracompartimentais apresentassem menor sobrevida, a localização não foi uma variável independente para a ocorrência de metástases.

\section{REFERÊNCIAS}

1. Enzinger FM, Weiss SW. Soft tissue tumors. 3rd ed. St. Louis: Mosby; c1995.

2. Ishihara HY, Jesus-Garcia Filho R, Korukian M, Ponte FM. Sarcoma de tecidos moles: fatores prognósticos. Rev Bras Ortop. 2004;39(11/12):637-47.

3. Engellau J. Prognostic factors in soft tissue sarcoma. Tissue microarray for immunostaining, the importance of wholetumor sections and time-dependence. Acta Orthop Scand Suppl. 2004;75(314):2p preceding table of contents-52, backcover.

4. Rydholm A. Prognostic factors in soft tissue sarcoma. Acta Orthop Scand Suppl. 1997;273:148-55. Review.

5. Coindre JM, Terrier P, Bui NB, Bonichon F, Collin F, Le Doussal V, et al. Prognostic factors in adult patients with locally controlled soft tissue sarcoma. A study of 546 patients from the French Federation of Cancer Centers Sarcoma Group. J Clin Oncol. 1996;14(3):869-77.

6. Strander H, Turesson I, Cavallin-Stahl E. A systematic overview of radiation therapy effects in soft tissue sarcomas. Acta Oncol. 2003;42(5-6):516-31.
Os três fatores histológicos estudados mostraram influência no desenvolvimento de metástases. O grau de malignidade é baseado na combinação da celularidade, atividade mitótica, pleomorfismo, anaplasia e necrose tumoral. A maioria dos estudos mostra que o alto grau de malignidade tem forte influência no desenvolvimento de metástases, sendo um dos mais importantes fatores independentes relacionados com a agressividade dos $\mathrm{STM}^{(2-5,7-8,10)}$. Entretanto, a falta de uma classificação uniforme, a incerteza para a classificação da importância de cada componente histológico, a subjetividade do examinador e o seu valor diferente nos diversos subtipos histológicos reduzem sua conformidade e sua reprodutibilidade ${ }^{(3)}$. Já a presença de necrose tumoral e invasão vascular detectadas pela histologia parecem ser fatores independentes para disseminação metastática; alguns sistemas já incluem esses achados como fatores para estadiamento ${ }^{(5,7,12)}$.

\section{CONCLUSÃO}

Os fatores associados à maior ocorrência de metástases nos STM são a localização extracompartimental do tumor, o seu tamanho, o grau de malignidade histológica, a presença de necrose e invasão vascular.

7. Gustafson P. Soft tissue sarcoma. Epidemiology and prognosis in 508 patients. Acta Orthop Scand Suppl. 1994;259:1-31.

8. Pisters PW, Pollock RE. Staging and prognostic factors in soft tissue sarcoma. Semin Radiat Oncol. 1999;9(4):307-14.

9. Enneking WF, Spanier SS, Goodman MA. A system for the surgical staging of musculoskeletal sarcoma. Clin Orthop Relat Res. 1980;(153):106-20.

10. Frustaci S, Gherlinzoni F, De Paoli A, Bonetti M, Azzarelli A, Comandone A, et al. Adjuvant chemotherapy for adult soft tissue sarcomas of the extremities and girdles: results of the Italian randomized cooperative trial. J Clin Oncol. 2001;19(5): 1238-47. Comment in: J Clin Oncol. 2001;19(5):1235-7.

11. Antman KH. Adjuvant therapy of sarcomas of soft tissue. Semin Oncol. 1997;24(5):556-60. Review.

12. Gustafson P, Akerman M, Avelgard TA, Coindre JM, Fletcher CD, Rydholm A, Willén H. Prognostic information in soft tissue sarcoma using tumor size, vascular invasion and microscopic tumor necrosis-the SIN-system. Eur J Cancer. 2003;39(11):1568-76. 
13. Cool P, Grimer R, Rees R. Surveillance in patients with sarcoma of the extremities. Eur J Surg Oncol. 2005;31(9): 1020-4.

14. Rüdiger HA, Beltrami G, Campanacci DA, Mela MM, Franchi A, Capanna R. Soft tissue sarcomas of the popliteal fossa: outcome and risk factors. Eur J Surg Oncol. 2007;33(4):512-7.

15. Leidinger B, Heyse T, Schuck A, Buerger H, Mommsen P, Bruening $\mathrm{T}$, et al. High incidence of metastatic disease in primary high grade and large extremity soft tissue sarcomas treated without chemotherapy. BMC Cancer. 2006;6:160.

16. Trovik CS, Bauer HC. Local recurrence of soft tissue sarcoma a risk factor for late metastases. 379 patients followed for 0.5-20 years. Acta Orthop Scand. 1994;65(5):553-8.
17. Spurrell EL, Fisher JC. Thomas JM, Judson IR. Prognostic factors in advanced synovial sarcoma: an analysis of 104 patients treated at the Royal Marsden Hospital. Ann Oncol. 2005;16(3):437-44.

18. Stojadinovic A, Jaques DP, Leung DH, Healey JH, Brennan MF. Amputation for recurrent soft tissue sarcoma of the extremity: indications and outcome. Ann Surg Oncol. 2001; 8(6):509-18.

19. Stojadinovic A, Leung DH, Allen P, Lewis JJ, Jaques DP, Brennan MF. Primary adult soft tissue sarcoma: timedependent influence of prognostic variables. J Clin Oncol. 2002;20(21):4344-52.

20. Rööser B, Attewell R, Rydholm A. The staging of soft-tissue sarcomas. Int Orthop. 1987;11(4):339-43. 